\title{
PERFIL LINGUÍSTICO, FAMILIAL E DO GÊNERO DE ESCOLARES COM DIAGNÓSTICO DE DISLEXIA DE UMA CLÍNICA ESCOLA
}

\author{
Linguistic, familial and gender profile of students diagnosed \\ with dyslexia of a school clinic
}

\author{
Nathane Sanches Marques Silva(1), Patrícia Abreu Pinheiro Crenitte ${ }^{(2)}$
}

\begin{abstract}
RESUMO
Objetivo: traçar o perfil dos pacientes com diagnóstico de dislexia quanto ao gênero, recorrência familial para distúrbios da comunicação ou dificuldade escolar, presença de alteração de linguagem oral e quanto à presença de alteração nas habilidades de memória de trabalho fonológica, memória visual e consciência fonológica na primeira avaliação fonoaudiológica, por meio da análise de prontuários de indivíduos. Métodos: foi realizado um estudo transversal retrospectivo por meio de análise de prontuários dos últimos dez anos, no período de 2001 a 2011, em que foram investigadas as histórias clínicas e a primeira avaliação interdisciplinar de pacientes diagnosticados com dislexia. Resultados: por meio de uma amostra de 23 prontuários, foi verificado que $82 \%$ dos pacientes pertencem a indivíduos do gênero masculino; $60,9 \%$ possuíam recorrência familial quanto à presença de familiares com distúrbios da comunicação ou dificuldades escolares; $47,8 \%$ dos escolares com diagnóstico de dislexia relataram sofrer de algum tipo de alteração de linguagem oral; $82,6 \%$ dos pesquisados possuíam alteração de memória de trabalho fonológica; $82,6 \%$ de consciência fonológica e $39,1 \%$ de memória visual. Conclusão: o perfil dos pacientes diagnosticados com dislexia, na Clínica Escola da instituição de origem, se caracteriza, preferencialmente pelo gênero masculino, pela presença de recorrência familial para distúrbios da comunicação ou dificuldades escolares e por apresentarem alteração de memória de trabalho fonológica e consciência fonológica durante a primeira avaliação fonoaudiológica.
\end{abstract}

DESCRITORES: Dislexia; Linguagem; Aprendizagem

\section{INTRODUÇÃO}

No processo de aprendizagem, a memória tem um papel fundamental, sendo necessária tanto a memória de curto quanto a de longo prazo. Assim, para o processo da escrita é preciso recuperar elementos léxicos por via fonológica ou indireta, podendo também ser por via ortográfica visual, ou seja, fazer uma evocação de conhecimentos adquiridos anteriormente e armazená-los por meio

(1) Faculdade de Odontologia de Bauru - FOB-USP, Bauru, São Paulo, Brasil.

(2) Faculdade de Odontologia de Bauru - FOB-USP, Bauru, São Paulo, Brasil.

Fonte de auxílio: Conselho Nacional de Apoio a Pesquisa

Conflito de interesses: inexistente da memória, tais como: grafemas, fonemas e/ou palavras. Sendo assim, a memória é necessária para a aprendizagem da escrita ${ }^{1}$. Para compreender a leitura é preciso ter integridade dos sistemas nervoso central e periférico, e para uma boa leitura também são necessários alguns pré-requisitos: atenção seletiva e sustentada, discriminação e percepção auditiva, memória de curto e longo prazo e, ainda, consciência fonológica² .

No processo de leitura também é essencial a memória visual. É pela discriminação visual, atenção e memória de armazenagem de informações gráficas que se faz o reconhecimento dos grafemas, sendo necessário um reconhecimento da ortografia gráfica, considerando que, o processamento visual é complementar ao processamento 
fonológico ${ }^{3}$. A memória visual é importante para o domínio de regras que vão determinar a grafia da palavra, sendo que os escolares em ditados visuais possuem melhor desempenho do que em ditados verbais, devido à requisição da memória de curto prazo ${ }^{4}$.

A dislexia, distúrbio específico de leitura, se caracteriza por um rendimento inferior ao esperado para a idade mental, nível sócio-econômico e instrução escolar, e pode afetar os processos de decodificação e compreensão da leitura ${ }^{5,6}$. Dessa forma, o indivíduo apresenta inteligência normal, distúrbio fonológico, falha nas habilidades semânticas, sintáticas e pragmáticas, tem a habilidade narrativa comprometida no processo de recontagem de histórias, dificuldade na fluência correta na habilidade de decodificação e soletração, além de ter alteração no processamento de informações auditivas e visuais ${ }^{7,8}$.

Foi evidenciada na literatura a recorrência familial para a dislexia ${ }^{9}$. Estudos mostram que a dislexia possui uma relevante incidência familial ${ }^{10}$, sendo este um importante fator de risco para o diagnóstico ${ }^{11,12}$. Foi verificada ainda, a alta prevalência desse diagnóstico em escolares do gênero masculino $0^{9,10}$.

Segundo alguns autores, durante o diagnóstico de crianças com dislexia observam-se algumas dificuldades relacionadas com a evocação da memória. Exemplo disso é que a criança é capaz de compreender a ideia central das leituras, mas não recorda os detalhes dos textos ${ }^{8}$. Outros estudos relatam que há evidências de alterações de funções executivas em crianças com dislexia, levando a um desempenho inferior na memória de trabalho, fluência verbal e de figuras, e controle inibitório, com capacidade parcialmente comprometida na resolução de problemas e habilidade para formação de conceitos sem comprometimento ${ }^{13}$. Escolares com dislexia, ainda, podem apresentar um desempenho inferior em tarefas de atenção visual sustentada e em componentes executivos de flexibilidade e inibição cognitiva, sem comprometimento no planejamento ${ }^{14}$.

Foi apontado que crianças disléxicas não apresentam menor inteligência do que crianças normais, e sim uma baixa capacidade de memória auditiva de curto prazo ou de memória de trabalho fonológica ${ }^{15}$. Alguns autores verificaram que as dificuldades em habilidades do processamento auditivo de atenção, codificação, organização e integração de informações auditivas são as que comprometem o uso das habilidades do processamento fonológico ${ }^{16}$. Assim, o processamento fonológico é fundamental para o bom desempenho de leitura ${ }^{17}$. Outro estudo expõe que as crianças com dislexia mostram ter dificuldades no processamento da memória de trabalho, além de alterações fonológicas e ortográficas ${ }^{18}$. A dificuldade central da dislexia relaciona-se com o processamento fonológico no que se refere à habilidade de consciência fonológica, memória de trabalho fonológica e nomeação verbal rápida que resultam em alterações na linguagem escrita ${ }^{19}$.

A consciência fonológica é uma habilidade do processamento fonológico explícito, que se refere à capacidade de reflexão sobre os sons das palavras e de manipulá-los. Tal habilidade prediz o desempenho de leitura e escrita. Verificou-se também que outras habilidades que predizem este desempenho são: a memória verbal de curto prazo e memória de longo prazo que fazem parte do processamento fonológico implícito ${ }^{20}$. Há casos de dislexia em que são observados problemas no processamento visual ${ }^{21}$. Existem indícios de que disfunções cerebrais no giro angular ou em sua volta, que são áreas envolvidas no armazenamento de palavras, devem levar à dislexia superficial ou diseidética.

São caracterizados três tipos de dislexia: a disfonética ou fonológica, derivada de uma inabilidade de aplicar a relação letra-som e é decorrente de um déficit no processamento fonológico ${ }^{6,21}$, resultado de dificuldade nas capacidades de percepção da fala, o que dificulta o desenvolvimento de consciência fonológica e, portanto, a segmentação fonêmica da fala que leva a problemas na de/codificação grafofonêmica necessária para o uso da rota fonológica, ou seja, prejudica a leitura de palavras não familiares ${ }^{22}$. A dislexia diseidética ou superficial, que é qualificada pela inaptidão de reconhecer palavras como um todo e resulta de um déficit no processamento visual e; por fim, a dislexia mista, que se caracteriza por dificuldades no processamento auditivo e visual ${ }^{6,23}$.

Assim, torna-se necessário o estudo das habilidades linguísticas, bem como o estudo da recorrência familial e da incidência quanto ao gênero do diagnóstico de dislexia em escolares.

Esta pesquisa tem como objetivo traçar o perfil dos pacientes com diagnóstico de dislexia quanto: ao gênero, recorrência familial para distúrbios da comunicação ou dificuldade escolar, presença de alteração de linguagem oral, presença de alteração nas habilidades de memória de trabalho fonológica, memória visual e consciência fonológica na avaliação fonoaudiológica.

\section{MÉTODOS}

As análises realizadas obedecem aos critérios do Comitê de Ética em Pesquisas da FOB-USP - Bauru, e este estudo só foi realizado após a 
aprovação do referido Comitê, sob o protocolo $n^{\circ}$ 192/2009.

A pesquisa foi realizada na Clínica de Fonoaudiologia da Faculdade de Odontologia de Bauru, da Universidade de São Paulo.

Foi realizado um estudo transversal retrospectivo por meio da análise de prontuários dos últimos dez anos, no período de 2001 a 2011, e foram investigadas as histórias clínicas (anamnese) e a primeira avaliação interdisciplinar de pacientes diagnosticados com dislexia. Os prontuários dos pacientes foram selecionados a partir de critério de inclusão; sendo o diagnóstico estabelecido de acordo com os critérios do DSM-IV-TR ${ }^{24}$ e CID-10 ${ }^{25}$ e comprovados pela avaliação interdisciplinar da Clínica de Fonoaudiologia da FOB/USP.

Os critérios de exclusão adotados foram: prontuários que não pertencem à área de atendimento de Leitura e Escrita, prontuários com avaliação interdisciplinar incompleta, com diagnóstico inconclusivo, e pertencentes a pacientes que apresentam outros problemas associados, como transtorno de déficit de atenção e hiperatividade.

Nas análises dos prontuários dos pacientes que se encontravam em atendimento no período estabelecido, foram coletados os seguintes dados: gênero, escolaridade, presença de alterações de linguagem, recorrência familial para distúrbios da comunicação ou dificuldades escolares, presença de alteração durante a primeira avaliação de memória de trabalho fonológica, memória visual e consciência fonológica.

Durante o estudo dos prontuários, observou-se que para a avaliação das habilidades de consciência fonológica, memória de trabalho fonológica e memória visual foram utilizados os seguintes instrumentos: "Perfil de Habilidades Fonológicas"26 para avaliar a consciência fonológica; a "Prova de Memória de Trabalho Fonológica - Não Palavras e Dígitos"27 para avaliar a memória de trabalho fonológica e o "Teste de Memória Visual da Bateria Neuropsicológica Luria-Nebraska"28 para avaliar a memória visual.

Os dados foram analisados utilizando-se tabelas, gráficos e estatística descritiva. O tratamento estatístico foi realizado a partir do emprego dos seguintes procedimentos: teste qui-quadrado e teste de proporções. O nível de significância adotado para este trabalho foi de $0,05(5 \%)$.

\section{RESULTADOS}

Foram analisados os prontuários referentes aos anos de 2001 a 2011, de escolares diagnosticados com dislexia pela Clínica de Fonoaudiologia da instituição de origem. Para isso, por meio do registro geral de 11.011 pacientes da clínica, foram selecionados os prontuários que tinham atendimento na área de Leitura e Escrita, ficando então, com uma amostra de 188 prontuários. Cada prontuário foi analisado minuciosamente seguindo os critérios de inclusão e exclusão descritos na metodologia e, assim, foi coletada uma amostra de 23 prontuários de pacientes com diagnóstico de dislexia pela Clínica de Fonoaudiologia.

Nos prontuários de pacientes com diagnóstico de dislexia, foi possível verificar na anamnese, que $82 \%$ dos pacientes pertencem a indivíduos do gênero masculino; que $60,9 \%$ da amostra possuía recorrência familial quanto a presença de familiares com distúrbios da comunicação ou dificuldades escolares, e $47,8 \%$ dos pais dos escolares com diagnóstico de dislexia relataram que seus filhos sofreram algum tipo de alteração da linguagem oral.

Por meio do teste qui-quadrado foi realizado a comparação dos dados de alteração de memória de trabalho fonológica, memória visual e consciência fonológica (comparação entre três porções), sendo encontrado o valor de $p=0,001$, mostrando diferença estatisticamente significante.

Os dados da primeira avaliação fonoaudiológica dos mesmos prontuários também foram analisados. A Figura 1, apresenta o gráfico com a porcentagem das alterações de memória de trabalho fonológica, de memória visual e das alterações combinadas das duas memórias. Por meio da análise estatística, foi possível verificar que houve diferença estatisticamente significante entre a incidência das duas memórias na amostra, visto que $p<0,05$. Por meio da análise deste gráfico é possível verificar que a memória de trabalho fonológica foi encontrada alterada em um número maior dos disléxicos pesquisados. Ainda permite a análise que a porcentagem das alterações combinadas de ambas as memórias tem o valor igual à porcentagem da alteração de memória visual, sendo que nos dados coletados, foi verificada que essa similaridade não é coincidência, já que todos os escolares com alteração de memória visual possuíam, também, alteração de memória de trabalho fonológica, enquanto que nem todos os escolares com alteração de memória de trabalho fonológica possuíam alteração de memória visual. 


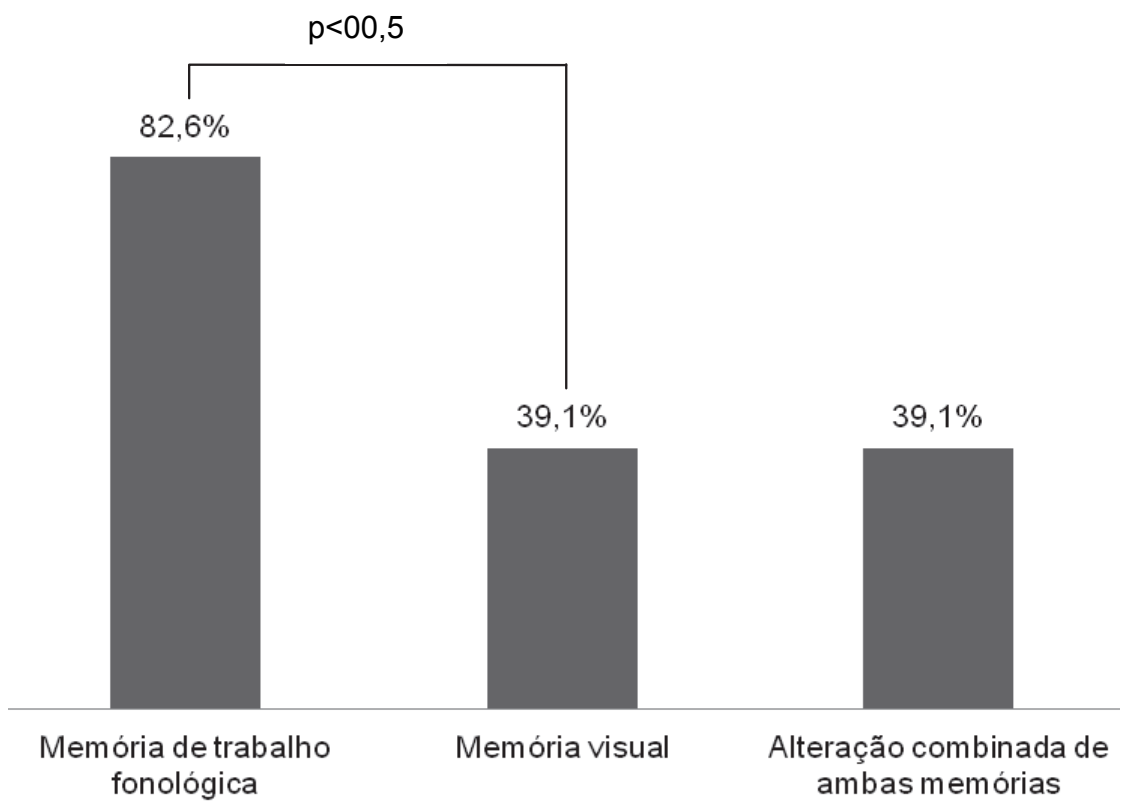

Teste qui-quadrado e teste proporções.

Figura 1 - Representação gráfica da porcentagem de disléxicos com alteração de memória de trabalho fonológica, memória visual e de das alterações combinadas das duas memórias

A Figura 2 apresenta o gráfico com a porcentagem das alterações de memória de trabalho fonológica, consciência fonológica e da alteração combinada das duas habilidades. Pela análise estatística, foi possível verificar que não houve diferença estatisticamente significante entre a incidência das duas habilidades na amostra, visto que $p>0,05$, Por meio deste gráfico é possível verificar que a consciência fonológica apresenta-se tão alterada nos indivíduos pesquisados quanto a memória de trabalho fonológica; porém nem todos os indivíduos com alteração de memória de trabalho fonológica apresentam alteração de consciência fonológica.

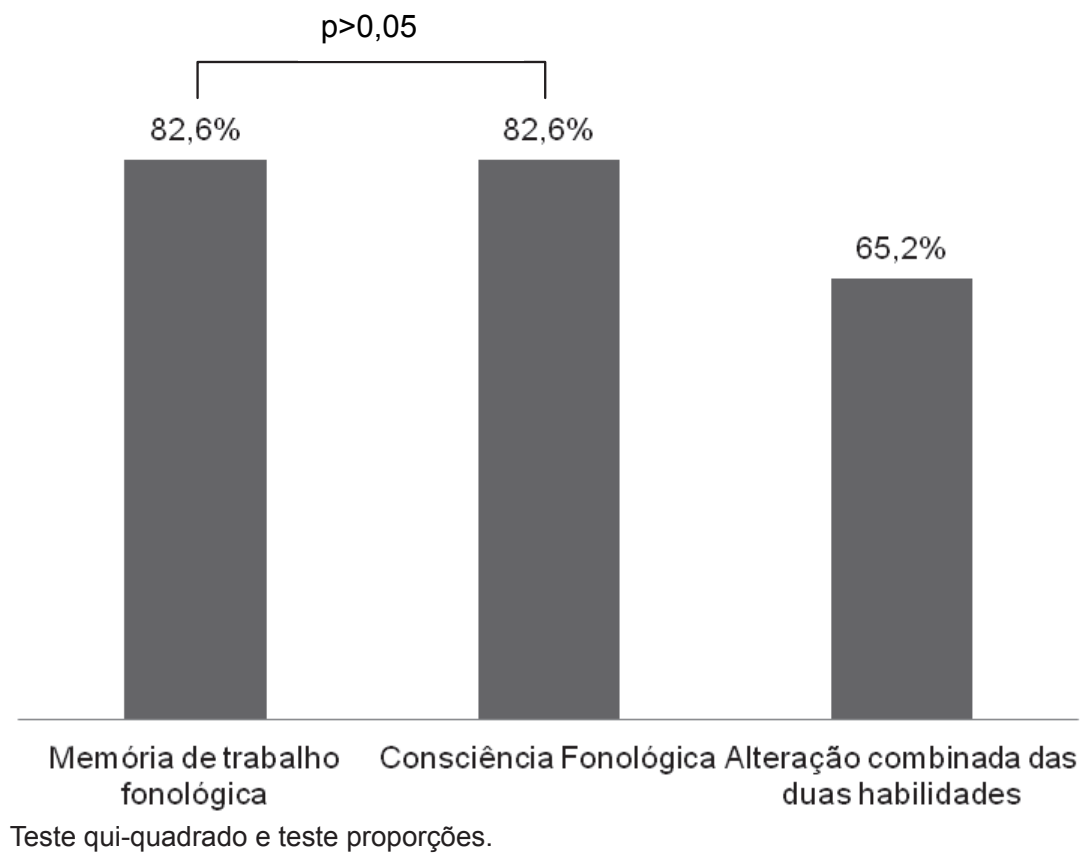

Figura 2 - Representação gráfica da porcentagem de disléxicos com alterações de memória de trabalho fonológica, consciência fonológica e da alteração combinada das duas habilidades 
AFigura 3 apresenta o gráfico com a porcentagem das alterações de memória visual, consciência fonológica e alteração combinada dessas duas habilidades. Nele é possível verificar que a consciência fonológica apresenta-se alterada em um maior número de indivíduos do que na memória visual. Utilizando-se o nível de significância de
0,05 , foi verificado que houve diferença significante entre a incidência das duas habilidades na amostra, visto que $p<0,05$. Ainda é possível verificar que os escolares com alteração de memória visual podem apresentar também a alteração de consciência fonológica.

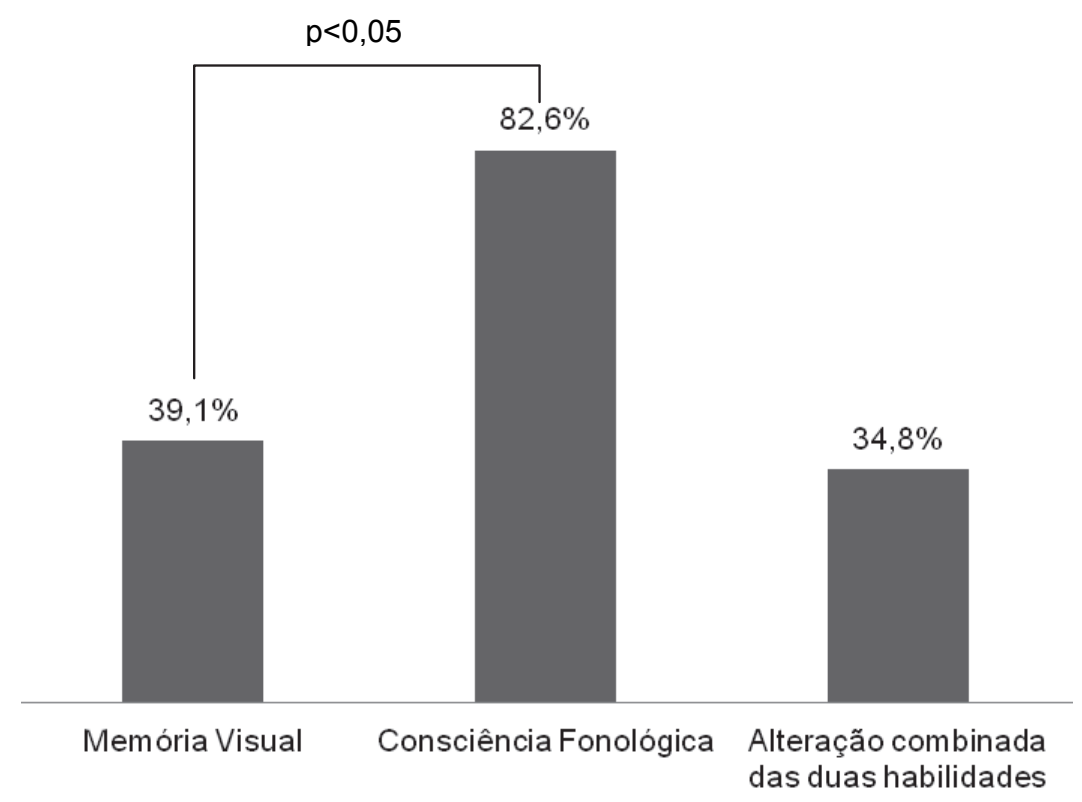

Teste qui-quadrado e teste proporções.

Figura 3 - Representação gráfica da porcentagem de disléxicos com alterações de memória visual, consciência fonológica e da alteração combinada das duas habilidades

\section{DISCUSSÃO}

Aincidência da dislexia na população geral ocorre em aproximadamente 10 a $15 \%{ }^{29}$, sendo que este fato pode justificar a pequena amostra da pesquisa. Foram excluídos os prontuários incompletos e aqueles que não tinham o diagnóstico estabelecido de acordo com os critérios do DSM-IV-TR ${ }^{24}$ e CID-10 25 . Os mesmos autores relataram que, assim, como outras alterações de linguagem, a dislexia, é mais prevalente no gênero masculino, porém dentro do mesmo levantamento bibliográfico, encontraram que o fato do gênero masculino ser mais prevalente, pode estar relacionado a um maior encaminhamento de professores, devido aos problemas de comportamento que geralmente acompanham mais os meninos do que as meninas com dificuldades escolares ${ }^{30}$. Outros estudos trazem que o cromossomo $\mathrm{X}$ aumenta o risco para dislexia, o que poderia explicar o porquê do sexo masculino ser mais afetado ou mais severamente afetado do que o feminino, levantando que a dislexia pode ser relacionada ao cromossomo $X^{10}$. Segundo outro autor, "os geneticistas do comportamento tem mostrado que há até $50 \%$ de probabilidade de um menino se tornar disléxico se seu pai for disléxico (cerca de $40 \%$ se sua mãe for afetada)"19. A prevalência do gênero masculino na amostra está de acordo com estudos pesquisados, porém não foi possível verificar se isto é decorrente do maior encaminhamento de meninos do que de meninas por parte dos professores.

Existem evidências que a dislexia é hereditária, sendo que não é herdada a dificuldade de leitura em si, mas os aspectos do processamento de linguagem ${ }^{19}$. Na amostra do presente estudo foi encontrada em mais da metade das anamneses a presença de recorrência familial, em que pelo menos um membro da família possuía dificuldades de aprendizado ou alteração de linguagem oral, o que está de acordo com o estudo que pesquisou uma amostra de familiares de disléxicos em que pelo menos um membro da família apresentava queixa ou dificuldade de aprendizado semelhante 
à dislexia, possibilitando dizer que a dislexia possui relevante incidência familial ${ }^{10}$.

Parte da amostra relatou alteração de linguagem oral. Verificou-se que a ocorrência de dificuldades anteriores na linguagem oral, como as dificuldades fonológicas e não as articulatórias podem ocasionar prejuízos no aprendizado da leitura e da escrita ${ }^{31}$. Outros autores já evidenciaram que os comprometimentos da linguagem oral podem ser um fator de risco para o processamento fonológico, e não somente os comprometimentos que permanecem depois dos seis anos de idade, mas também aqueles que já foram resolvidos podem interferir na alfabetização de uma criança. Ainda demonstram que as dificuldades de linguagem oral decorrentes de alterações no processamento fonológico são estabelecidas como um fator de risco para a dislexia ${ }^{32}$.

Outro estudo levantou a preocupação do diagnóstico e da prática diferenciada do professor com o aluno, mas não somente no ensino fundamental, mas também no ensino médio, além de apontar para o fato de que quando não ocorre o diagnóstico e a intervenção adequada, o fracasso escolar pode levar o aluno a reprovação ou ainda à evasão escolar, chegando a acarretar até problemas de comportamento no mercado de trabalho. Por isso, o diagnóstico e a intervenção precoce são fundamentais para minimizar as dificuldades que podem ocorrer na vida acadêmica dos disléxicos ${ }^{33}$. A mesma autora levantou a importância da criação de políticas púbicas para possibilitar a identificação dos disléxicos nas escolas públicas, além de permitir o suporte escolar adequado para esta população ${ }^{33}$.

Como já observado, na revisão bibliográfica realizada para esta pesquisa, há uma forte relação entre alteração de consciência fonológica e alteração de memória de trabalho fonológica em indivíduos com dislexia e este dado foi verificado também na amostra coletada. Por meio de levantamento bibliográfico, autores apontaram que distúrbios de memória de curto e longo prazo, tanto de memória auditiva e visual, podem ser encontrados em crianças com dislexia ${ }^{6}$. Estudos evidenciam que o déficit fonológico pode ser observado por meio de atrasos de habilidades de memória de trabalho fonológica e consciência fonológica que podem perdurar até a vida adulta ${ }^{19}$. A literatura mostra também, que maus leitores possuem desempenho significantemente menor que bons leitores em tarefas de escrita, consciência fonológica, vocabulário, memória de trabalho fonológica de curto prazo e memória visual, levantando que os processos envolvidos na leitura e na escrita estão fortemente relacionados ao processamento fonológico, incluindo a consciência fonológica e a memória de trabalho fonológica; sendo estas habilidades pré-requisitos para a aquisição da linguagem escrita ${ }^{22}$. Há também relatos na literatura, de que existem evidências que crianças com dislexia possam apresentar alterações das funções executivas podendo levar a um desempenho inferior em algumas habilidades, dentre elas a habilidade de memória de trabalho fonológica ${ }^{13}$. Outro estudo relatou que disléxicos apresentam alteração de consciência fonológica e do processamento temporal auditivo, cujo desempenho pode ter sido interferido pelas habilidades fonológicas, cognitivas e também pela memória de trabalho fonológica ${ }^{34}$. Tais alterações podem resultar de dificuldades no processamento da memória de trabalho, além de alterações fonológicas e ortográficas $^{19}$. Os dados encontrados na análise dos prontuários mostraram-se compatíveis com os dados publicados na literatura, os quais afirmam que as alterações no processamento fonológico estão presentes em indivíduos com dislexia, uma vez que os mesmos apresentam alterações de memória de trabalho fonológica e de consciência fonológica, e estas habilidades encontram-se alteradas em uma parcela significante dos indivíduos pesquisados.

Nos dados, foi ainda verificado que a prevalência de alteração de memória visual é baixa quando comparada às demais habilidades pesquisadas, porém sua presença reforça a ideia que a memória visual pode ser uma exceção dentro das habilidades de processamento visual na correlação com o desempenho de leitura e escrita ${ }^{35}$. Os escolares com dislexia podem apresentar alterações auditiva e visual, na orientação e atenção espacial, que pode gerar uma dificuldade para a seleção e percepção dos estímulos, sendo que tais alterações podem distorcer o desenvolvimento das representações fonológicas e ortográficas ${ }^{36}$. Dificuldades no processamento visual, como alteração de memória visual, podem acentuar as dificuldades apresentadas por disléxicos ${ }^{37}$. Outro estudo mostra a importância desta habilidade para o domínio de regras que vão determinar a grafia da palavra ${ }^{4}$.

Dessa forma, o presente estudo identifica a importância do diagnóstico adequado, levando em consideração o perfil linguístico, familial e de gênero dos escolares com dislexia. Ainda confirma a evidência encontrada na literatura de que existe a necessidade de fonoaudiólogos junto com os professores utilizarem programas de intervenção com base fonológica, com enfoque na memória de trabalho fonológica e visual, e na relação letra-som, a fim de identificar e intervir precocemente nos sinais da dislexia ${ }^{38}$. 


\section{CONCLUSÕES}

Os dados obtidos neste estudo, por meio da análise de prontuários de indivíduos diagnosticados com dislexia, mostraram-se compatíveis com os dados publicados na literatura, mesmo possuindo uma pequena amostra. Os resultados da pesquisa permitem concluir que o perfil dos pacientes diagnosticados com dislexia pela Clínica Escola de Fonoaudiologia da instituição de origem se caracteriza, preferencialmente, pelo gênero masculino, presença de recorrência familial para distúrbios da comunicação ou dificuldades escolares, e por apresentarem alteração de memória de trabalho fonológica e consciência fonológica durante a primeira avaliação fonoaudiológica.

\begin{abstract}
Purpose: to define the profile of patients diagnosed with Dyslexia in terms of gender, familial recurrence for communication disorders or difficulties at school, presence of oral language disorders and the presence of changes in the skills of phonological working memory, visual memory and phonological awareness in the first clinical assessment, through analysis of charts of individuals. Methods: we conducted a retrospective study cross-sectional study through analysis of charts of the last ten years, from 2001 to 2011, in which we investigated the clinical histories and interdisciplinary review of patients diagnosed with Dyslexia. Result: through a sample of 23 medical records, it was found that $82 \%$ of patients belong to male subjects; $60.9 \%$ had familial recurrence for the presence of relatives with communication disorders or learning difficulties; $47.8 \%$ of children diagnosed with dyslexia reported suffering from some type of change in oral language; $82.6 \%$ of surveyed possessed alteration of phonological working memory, phonological awareness $82.6 \%$ and $39.1 \%$ of visual memory. Conclusion: it was possible to verify that the profile of patients diagnosed with dyslexia, Clinical School at the home institution, is characterized, preferentially by male gender, the presence of familial recurrence for communication disorders or learning difficulties, and present a modification of phonological working memory and phonological awareness during the first clinical assessment.
\end{abstract}

KEYWORDS: Dyslexia; Language; Learning

\section{REFERÊNCIAS}

1. Souza ARM, Sisto FF. Dificuldade de aprendizagem em escrita, memória e contradições. Psicol. Esc. Educ.2001;5(2):39-47.

2. Sauer L, Pereira LD, Ciasca SM, Pestun M, Guerreiro MM. Processamento auditivo e SPECT em crianças com dislexia. Arq. Neuro-Psiquiatr. 2006;64(1):108-11.

3. Pino M, Bravo L. La Memoria Visual como Predictor del Aprendizaje de la Lectura. Psykhe. 2005;14(1):47-53.

4. Barbosa PMF, Bernardes NGB, Misorelli MI, Chiappetta ALML. Relação da memória visual com o desempenho ortográfico de crianças de $2^{a}$ e $3^{\text {a }}$ séries do ensino fundamental. Rev. CEFAC. 2010;12(4):598-607.

5. Capellini SA. Distúrbios de aprendizagem versus dislexia. In: Ferreira LP, Befi-Lopes DM, Limongi SCO. Tratado de Fonoaudiologia, São Paulo: Roca; 2004. p. 862-76.
6. Olmino EFN, Santana MSR. Dislexia: definição e caracterização. In: SCENCULT - Simpósio Cientifico Cultural, 6, 2011, Mato Grosso do Sul, Anais. Mato Grosso do Sul: SCENCULT, 2011.

7. Germano GD, Capellini SA. Eficácia do programa de remediação auditivo-visual computadorizado em escolares com dislexia. Pró-Fono Rev. Atual. Cient. 2008;20(4):237-42.

8. Deuschle VP, Cechella AC. O déficit em consciência fonológica e sua relação com a dislexia: diagnóstico e intervenção. Rev. CEFAC. 2009;12(2):194-200.

9. Prado DGA, Armigliato ME, Salgado C, LopesHerrera SA, Crenitte PAP. Dislexia e distúrbio de aprendizagem: histórico famílial. Distúrb Comum. 2012;24(1):77-84.

10. Capellini SA, Padula NAMR, Santos LCA, Lourenceti MD, Carrenho EH, Ribeiro LA. Desempenho em consciência fonológica, memória operacional, leitura e escrita na dislexia familial. Pró-Fono Rev. de Atual. Cient. 2007;19(4):374-80. 
11. Scarborough HS. Very early language deficits in dyslexic children. Child Dev. 1990;61(6):1728-43.

12. Meng $H$, Smith SD, Hager $K$, Held M, Liu $\mathrm{J}$, Olson RK et al. DCDC2 is associated with reading disabilities and modulates neuronal development in the brain. Proc. Nat. Acad. Sci, 2005;102(47):17053-8.

13. Dias NM, Menezes A, Seabra AG. Alterações das funções executivas em crianças e adolescentes. Estudos interdisciplinares em Psicologia. 2010;1:80-95.

14. Lima RF, Travaini PP, Salgado-Azoni CA, Ciasca, SM. Atención sostenida visual y funciones ejecutivas en niños con dislexia de desarrollo. Anales de psicología 2012;28(1):66-70.

15. Furbeta, TDC, Felippe ACN. Simplified auditory processing test and difficulties in reading and spelling. Pró-Fono Rev Atual Cient. 2005;17(1):11-8. 16. Pinheiro $\mathrm{FH}$, Germano GD, Capellini SA. Caracterização do desempenho em habilidades auditivas e fonológicas em escolares com dislexia do desenvolvimento. Tecer. 2011;4:1-13.

17. Jiménez JE, Guzmán R, Ortiz R, Díaz A, Estévez A, García E, et al. Validez discriminante de La Batería Multemedia Sicole-R-Primaria para la evaluación de procesos cognitivos asociados a la dislexia. Revista de Investigación Educativa. 2009;27(1):49-71.

18. Capellini SA, Germano GD, Cardoso ACV. Relação entre habilidades auditivas e fonológicas em crianças com dislexia do desenvolvimento. Psicol. Esc. Educ. (Impr.). 2008;12(1):235-51.

19. Snowling M. Dislexia desenvolvimental: uma introdução e visão teórica geral. In: Snowling M, Stackhouse J. Dislexia Fala e Linguagem - Um manual do profissional. Artmed; 2004. p. 11-21.

20. Defior S, Serrano F. Procesos fonológicos explícitos e implícitos, lectura y dislexia. Revista Neuropsicología, Neuropsiquiatría y Neurociencias, Colombia. 2011;11(1):79-94.

21. Pinheiro ÂMV. Dislexia do desenvolvimento: perspectivas cognitivo-neuropsicológicas. Athos \& Ethos, 2002;2:1-14.

22. Capovilla AGS, Capovilla FC, Suiter I. Processamento cognitivo em crianças com e sem dificuldades de leitura. Psicol. Estud. 2004;9(3):449-58.

23. Boder E. Developmental dyslexia: a diagnostic approach based on three atypical reading-spelling patterns. Developmental Medicine and Child Neurology. 1973;15:663-973.

24. American PsychiatricAssociation (APA). DSM-IV: Manual Diagnóstico e Estatístico de Transtornos mentais. 4 ed. rev trad. Caludia Dornelles. Porto Alegre: Artes Médicas; 2002.

25. Organização Mundial da Saude. Classificação de Transtornos mentais e de comportamento da CID-10: descrições clinicas e diretrizes diagnósticas. Porto Alegre: Editora Artes Médicas; 1993.

26. Alvarez AMMA, Carvalho IAM, Caetano AL. Perfil de habilidades fonológicas. São Paulo: Via Lettera, 1998.

27. Hage SRV, Grivol MA. Desempenho de crianças normais falantes do português em prova de memória de trabalho fonológica. Cad.Comun. Ling. 2009;1:11-22.

28. Lima RF, Mello RJL, Massoni I, Riechi TIJS, Ciasca SM. Teste Luria Nebraska-C. Campinas: Departamento de Neurologia, Faculdade de Ciências Médicas, Universidade de Campinas; 2005.

29. lanhez ME, Nico, M.A. Nem sempre é o que parece: como enfrentar a dislexia e os fracassos escolares. São Paulo: Alegro;2002.

30. Salles JF, Parente MAMP, Machado SS. As dislexias de desenvolvimento: aspectos neuropsicológicos e cognitivos. Interações. 2004;9(17):109-32.

31. Schirmer CR, Fontoura DR, Nunes ML. Distúrbios da aquisição da linguagem e da aprendizagem. J. Pediatr. 2004;80(2):95-103.

32. Tannock R. Desenvolvimento da linguagem e alfabetização: Comentários sobre Beitchman e Cohen. In: Tremblay RE, Boivin M, Peters RDEV, eds. Enciclopédia sobre o Desenvolvimento na Primeira Infância [on-line]. Montreal, Quebec: Centre of Excellence for Early Childhood Development; 2011 [acesso em 10 jul. 2011]:1-5. Disponível em: http://www.enciclopedia-crianca.com/documents/ TannockPRTxp1.pdf.

33. Souza EC. O disléxico com sujeito do processo ensino-aprendizagem. Contrapontos. 2008;8(3):423-32.

34. Murphy CFB, Schochat E. Correlações entre leitura, consciência fonológica e processamento temporal auditivo. Pró- Pró-Fono Rev Atual Cient. 2009;21(1):8-13.

35. Capovilla AGS, Gütschow CRD, Capovilla, FC. Habilidades cognitivas que predizem competência de leitura e escrita. Psicol. Teor. Prat. 2004;6(2):13-26.

36. Facoetti A, Lorusso ML, Paganoni P, Cattaneo C, Galli R, Umiltà C et al. Auditory and visual automatic attention deficits in developmental dyslexia. Cognitive Brain Research. 2003;16(2):185-91. 
37. Machado AC, Capellini SA. Caracterização do desempenho de crianças com dislexia do desenvolvimento em tarefas de escrita. Rev. Bras. Cresc. e Desenv. Hum. 2011;21(1):132-8.
38. Fukuda MTM, Capellini SA. Treinamento de habilidades fonológicas e correspondência grafema-fonema em crianças de risco para dislexia. Rev CEFAC. 2011;13(2):227-35.

http://dx.doi.org/10.1590/1982-0216201414612

Recebido em: 04/07/2012

Aceito em: 18/01/2013

Endereço para correspondência:

Nathane Sanches Marques Silva

Rua Professor Gerson Rodrigues, 7-51

Bauru - SP

CEP: 17012-535

E-mail: nathane.sm@gmail.com 